\title{
Devising a Training Method for Assembly Work by Employing Disassembly
}

\author{
Osamu Ichikizaki* \\ Faculty of Science and Technology, Keio University, Yokohama, Japan \\ Ryou Kubota, Toshikazu Komori, Toshiyuki Matsumoto* \\ Department of Industrial and Systems Engineering, Aoyama Gakuin University, Sagamihara, Japan \\ Akihiro Erikawa \\ Otomo Seisakusho Co., Ltd., Tokyo, Japan
}

(Received: January 20, 2013 / Revised: July 9, 2013 / Accepted: October 29, 2013)

\begin{abstract}
Efficiency in work training is a perennial issue due to high-diversity low-volume production, particularly for manufacturers producing office machines which are manually assembled by workers. To reduce the training cost, parts used in training are usually reused; a trainer disassembles a product assembled by a worker in training. This paper proposes a training method that employs disassembly usually performed by a trainer. This method assigns both assembly and disassembly to a worker in training, in contrast to the conventional method. The effectiveness of the proposed method is experimentally discussed in terms of learning assembly motions and work procedure at each learning stage, namely, "undergoing learning," "immediately after learning," and "seven days after learning." The effectiveness of the training method is confirmed. The method improves the stability of work procedure recollection immediately after training. Furthermore, at seven days after training, it improves retention of the assembly motions and work procedure, and also promotes and maintains memory related to product structure.
\end{abstract}

Keywords: Assembly, Disassembly, Motion, Work Procedure, Learning Process

*Corresponding Author, E-mail: ichiki@ae.keio.ac.jp, matsumoto@ise.aoyama.ac.jp

\section{INTRODUCTION}

In recent years, products, such as office machines -which are often manually assembled by workers - are being increasingly manufactured through high-diversity low-volume production. Due to the greater variety of product types and the increased frequency of changes in product specifications and model upgrades, more work details should be learned. As a result, opportunities for training are increasing. Due to the effects of global cost competition, more reduction of labor costs is required, and the widespread worker outsourcing and migration also have an effect in increasing training opportunities. Furthermore, due to the reduction in volume, manufac- turing systems are shifting from the line to the cell type, and individual workers have to cover many types of work. This increases training time. Since training opportunities and training time increase in this way, many manufacturing factories face the issue of improving the efficiency of work training.

In addition, in recent years the cost of work training has also become an issue. When products were manufactured in high-volume in the line production system, as they were in the past, only a few types of work were covered by a single worker. Therefore, it was possible to provide training in basic skills beforehand while offline, and then trainers could provide online training in essential skills, while supporting those workers. It was also 
possible to use product parts for training, so there was almost no need to prepare parts separately for training. However, as noted above, there have been increases in the frequency of training and in the types of work handled by a single worker, and thus it has become difficult to conduct training combining offline and online approaches. Therefore, it has become necessary to procure parts for training, but in an environment where cost reduction is needed more than ever before, it is not easy to procure parts in sufficient quantities. For example, in order to conserve parts for training at a certain photo developing machine plant, the trainer disassembles semifinished products assembled by the workers undergoing training, and the parts are reused as parts for training. Many office machine products can be disassembled, for reasons, such as maintenance, and plants which conduct work training using this same method are not unusual.

When carrying out disassembly work during training, worker memory of product structure can be enhanced by having the workers undergoing training do the disassembly indicated above instead of the trainer. On the other hand, there may be a disadvantage in that workers are asked to do work other than assembly, and thus the situation must be comprehensively examined.

For the above reasons, this study assumes a manually assembled product, and proposes a work training method that employs disassembly, which is needed when there are constraints on the number of parts that can be used for training. The purpose is to experimentally verify the effectiveness of this method.

\section{POSITIONING OF THIS STUDY RELA- TIVE TO RELATED RESEARCH}

The typical training method - in which workers are repeatedly instructed to only carry out assembly work, and the trainer disassembles the products assembled by the workers - will be referred to as "assembly training." On the other hand, the training method proposed in this study - in which workers are instructed to perform disassembly work as well-will be referred to as "assembly/disassembly training."

There have been many studies on work training which aim to reduce work time in aircraft manufacturing (Alchian, 1963; Belvis et al., 1970; Fukuda and Ohkubo, 1993; Hirschmann, 1964; Seibel, 1964; Wright, 1936). Since then, research has increasingly focused not only on work time as a result, but also on analysis of the process. In assembly work involving a great deal of manual assembly, reduction of work time can be achieved by dividing learning into two categories: learning motions and learning work procedures. Research on learning motions includes studies showing that learning is promoted when teaching is done by providing feedback on differences in work between beginners and experts as physical quantities which can be perceived by the operator using an operation device, or when teaching is done visually using a monitor (Shida et al., 2003; Suenaga, 1998; Suenaga and Ihara, 1995). Research on learning work procedures includes studies focused on work which does not require difficult motions in which effective learning methods were proposed by evaluating learning status based on the level of work elements (Jittachalothorn et al., 1996, 1997; Jittachalothorn, 1998, 2000); and studies on effective sequential presentation via a PC using text, still images and movies (Shida et al., 2003; Shida, 2005). Learning of work procedures has also been examined from the perspective of forgetting and mistakes, and research in this area includes studies relating to proposal of models for analysis of learning process until learning is achieved (Ichikizaki et al., 2009), and studies showing that certain types of mistakes during training promote learning (Ichikizaki, 2006; Ichikizaki et al., 2010).

As indicated above, there have been many studies on work training, but no research was found concerning the assembly/disassembly training which is the subject of this study, and its effectiveness. Therefore, this study evaluates the work training from the perspectives of learning motions and learning work procedures. Regarding learning work procedures in particular, this study focuses on memory of product structure, which can potentially be promoted by the assembly/disassembly training. The assembly training and the assembly/disassembly training are experimentally compared based on the above perspectives, and an attempt is made to show the effectiveness of the assembly/disassembly training.

\section{EXPERIMENTAL WORK}

In this study, the following requirements were established for selecting experimental work to carry out an experimental comparison of the assembly training and the assembly/disassembly training. The third and fourth requirements are based on the survey results on semifinished products at a certain plant for photo developing machines.

- Assuming assembly work in the cell production system, the product must be comprised of 30 to 40 parts.

- Disassembly must be possible, as with most types of

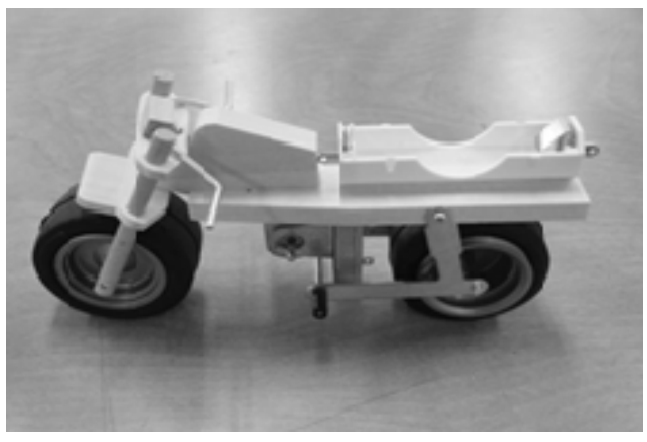

Figure 1. Experimental product: mini-bike. 
Table 1. Part characteristics

\begin{tabular}{|c|c|c|c|c|c|}
\hline No. & Part name & Contact relationship & No. & Part name & Contact relationship \\
\hline 1 & Tank and frame & $\underline{\mathbf{2}}, \mathbf{4}, 8,14,15,17,18,22,23$ & 15 & Motor case & $1,13,14,16,19,20$ \\
\hline 2 & Switch case A & $3,4, \underline{\mathbf{6}}, \mathbf{7}, 12,13$ & 16 & Motor & 15 \\
\hline 3 & Switch case B & $2,4, \overline{5}, \overline{7}$ & 17 & Fender & $1,22,28$ \\
\hline 4 & Switch case $\mathrm{C}$ & $2,3, \underline{\mathbf{5}}, 6,7,13$ & 18 & Handle & 1,23 \\
\hline 5 & Switch fitting A & 3,4 & 19 & $2 \mathrm{~mm}$ Shaft & $15,20,21$ \\
\hline 6 & Switch fitting B & $\underline{\mathbf{2}}, 4$ & 20 & Swing arm & $1,14,15,19,21,26,27$ \\
\hline 7 & Switch fitting $\mathrm{C}$ & $\underline{\mathbf{2}}, 4$ & 21 & Rubber pipe (L) & 19,20 \\
\hline 8 & Battery box & $9,10,11,14$ & 22 & Rubber pipe (S) & 17,23 \\
\hline 9 & Metal fitting A & $8,10,11$ & 23 & Rivet & $1,17,18,22$ \\
\hline 10 & Metal fitting B & 8,9 & 24 & Front shaft & 25,28 \\
\hline 11 & Metal fitting $\mathrm{C}$ & 8,9 & 25 & Front tire & 24 \\
\hline 12 & Nut & 2,13 & 26 & Rear shaft & 20,27 \\
\hline 13 & Round screw & $2,4,12,15$ & 27 & Rear tire & 26 \\
\hline 14 & Tapping screw & $1,8,15,20$ & 28 & Front fork & $17,18,24$ \\
\hline
\end{tabular}

Table 2. The experiments and the evaluation measures

\begin{tabular}{lcccc}
\hline \multirow{2}{*}{ Experiment } & \multicolumn{2}{c}{ Motion } & \multicolumn{2}{c}{ Work procedure } \\
\cline { 2 - 6 } & Speed & Accuracy & Speed & Accuracy \\
\hline $\begin{array}{c}\text { Grouping experiment } \\
\text { Screw tightening test } \\
\text { Card arrangement memory test }\end{array}$ & Work time & $\begin{array}{c}\text { Total distance } \\
\text { of the gaps }\end{array}$ & - & - \\
Main experiment* \\
$\begin{array}{c}\text { Undergoing training } \\
\text { Training experiments }\end{array}$
\end{tabular}

* Assembly training and Assembly/disassembly training.

office machine products.

- There must be four types of assembly structure: fitting, connection, mounting, and screw fastening.

- There must be five types of positioning (Fujita, 1978) when mounting parts: fitting, aligning, inserting, placing, and adjusting.

The wooden small motorcycle model shown in Figure 1 (TAMIYA 70095, referred to as the "mini-bike") below was selected as a product satisfying the above requirements, and adopted as the experimental work for this study. The mini-bike is comprised of 39 parts (28 types). Table 1 shows the part characteristics, including the part names and contact relationships of each part.
The parts in the table whose numbers are underlined are parts which are touching but not mounted.

\section{DESIGN OF EXPERIMENTS}

The two types of training were experimentally compared for the above experimental work, and the experiment shown in Table 2 was conducted to show the effectiveness of the assembly/disassembly training.

First, a grouping experiment (screw tightening test and card arrangement memory test) was performed for assigning subjects to groups with the same ability for the assembly training and the assembly/disassembly training. 
In the grouping experiment, the motion and memory ability of subjects were both evaluated in terms of speed and accuracy.

Next, experiments for each type of training (training experiments) were conducted on the assigned subjects. Immediately after the training experiments, an experiment was conducted to confirm the degree of learning of the work procedure (work learning confirmation test).

In addition, seven days after the training, as a posttraining experiment, 2 tests were conducted to investigate retention of the assembly motions and work procedures (work retention confirmation test) and to investigate retention of memory relating to the product structure (structure retention confirmation test) for a supplementary experiment on retention of work procedures,.

The following describes the details of each of the above experiments.

\subsection{Grouping Experiment}

A screw tightening test was conducted to evaluate motion capabilities (speed and accuracy), and a card arrangement memory test was conducted to evaluate memory (recollection) capabilities (speed and accuracy). Details on each test are indicated below.

\subsubsection{Screw tightening test}

The screw tightening test used a wooden panel $(22.5 \times 4.5 \times 1.0 \mathrm{~cm})$ with 10 pilot holes $(0.2 \mathrm{~cm})$ drilled at equal intervals $(5 \mathrm{~cm}), 10$ tapping screws $(\mathrm{M} 1 \times 5$ size $)$, and a Phillips-head screwdriver. The subjects were asked to use the Phillips-head screwdriver to tighten the 10 tapping screws into the 10 holes in the wood panel. To ensure that the experiment was carried out under the same conditions for all subjects, wood panels and tapping screws were provided for each subject, and all subjects used the same Phillips-head screwdriver. In addition, the content of instructions prior to the experiment (order of tightening tapping screws, etc.) was made the same in all cases. Figure 2 shows the layout of the screw tightening test. The wood panel was fastened to a table; the 10 tapping screws were placed in a parts box positioned on the far side of the wood panel; and the Phillips-head screwdriver was placed on the right side (or the left side if the subject was left-handed).

Subject motion speed was evaluated based on work time, and motion accuracy was evaluated based on the total distance of the gaps between the bottom surface of the screw head and the wood panel (which arise when screws are not fastened vertically with respect to the wood panel). The total distance was the total gap measured for all 10 screws after tightening. Work time was measured with a stopwatch, and the total gap distance was measured with a thickness gauge.

\subsubsection{Card arrangement memory test}

A total of 8 cards, combining the symbols (circle,

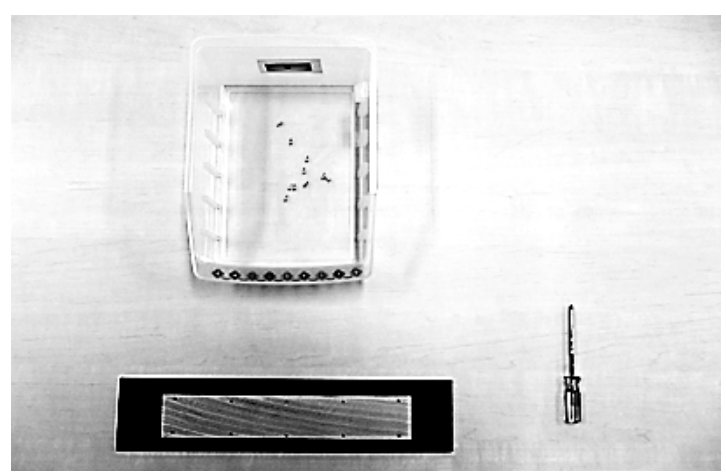

Figure 2. Layout for the screw tightening test.

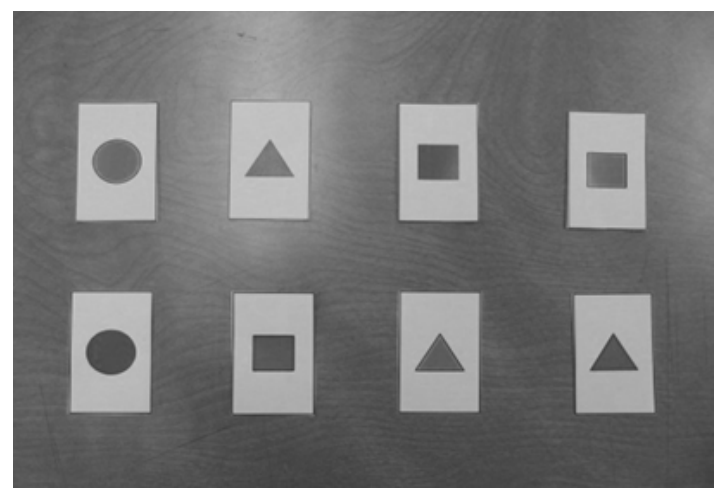

Figure 3. Card arrangement of the card arrangement memory test.

square, and triangle) and the colors (red, yellow, and blue) were used for the card arrangement memory test. The subjects memorized an arrangement of 8 cards for at most 30 seconds, and then later wrote down the memorized card arrangement onto a specified sheet of paper within 3 minutes at most. To ensure that the experiment was carried out under the same conditions for all subjects, the card arrangement was made the same in all cases. Figure 3 shows the card arrangement for this test.

Subject memory (recollection) speed was evaluated based on recollection time (time needed to recollect the card arrangement), and memory accuracy was evaluated based on the number of correctly recorded card positions and colors (the position and color for one card are individually checked, so the maximum number is 16). Card arrangement recollection time was measured using a stopwatch.

\subsection{Training Experiments}

In the main experiment, effectiveness of the assembly/disassembly training was verified by experimentally comparing the two alternatives: the assembly training and the assembly/disassembly training.

The experiments on the two types of training for the experimental work were conducted using the following procedures. In both experiments, the purpose was to 
evaluate the learning process for the motions and the work procedure in training. The speed of motion learning in training was evaluated based on the work time of each cycle, and the accuracy of learning the work procedure was evaluated based on the number of mistakes. It is difficult to directly evaluate the accuracy of motion learning and the speed of work procedure learning, and thus these were evaluated indirectly based on the number of mistakes, and the work time of each cycle.

\subsubsection{Assembly training experiment}

Mini-bike assembly work was repeated 10 times. The subjects performed the work according to the previously designed work procedure shown in Figure 4. One of the work elements of this work procedure is the mounting of each part. The instructions for each work element include a photo showing the nature of the work (state after mounting), the work procedure, and (in cases where necessary) standards and points on quality.

A3 size paper was placed in front of the subject, and parts were arranged there in the sequence specified in the work procedure (Figure 5). The subject assembled the mini-bike while reading the work procedure and following its sequence. After that, an experiment assistant disassembled the mini-bike assembled by the subject, and placed the parts on the paper indicated above. This cycle was repeated 10 times, and then the experiment was finished.

Prior to the experiment, all of the subjects were given the following instructions besides the purpose of this experiment, the state of starting the experiment, and the state of finishing the experiment. Firstly, when a subject reads the work procedure and still does not understand, the subject is allowed to ask a question, but only for the first mini-bike. Secondly, when a subject has any questions, the subject should not think about it, and immediately raise the hand and ask questions instead. Thirdly, a subject is expected to try to assemble the second and subsequent mini-bikes while looking at the work procedure as little as possible, but refresh the memory by looking at the work procedure when the subject forgets the steps.

\subsubsection{Assembly/disassembly training experiment}

Mini-bike assembly and disassembly work were repeated 10 times. The subjects performed assembly work according to the work procedure, just as in the assembly training experiment, and then performed disassembly work according to the disassembly procedure prepared in the same format.

A3 size paper was placed in front of the subject, and parts were arranged there in the sequence specified in the work procedure. However, to clearly indicate the place to return the part during disassembly work, two of each part were placed on the paper (Figure 6). The subject assembled the mini-bike while reading the work procedure and following its sequence. After that, the parts were arranged on the above paper according to the

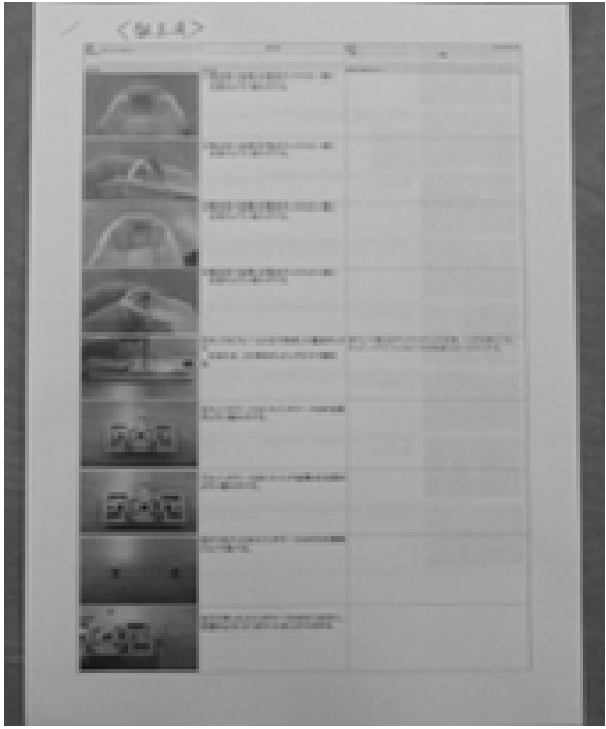

Figure 4. Example of the work procedure.

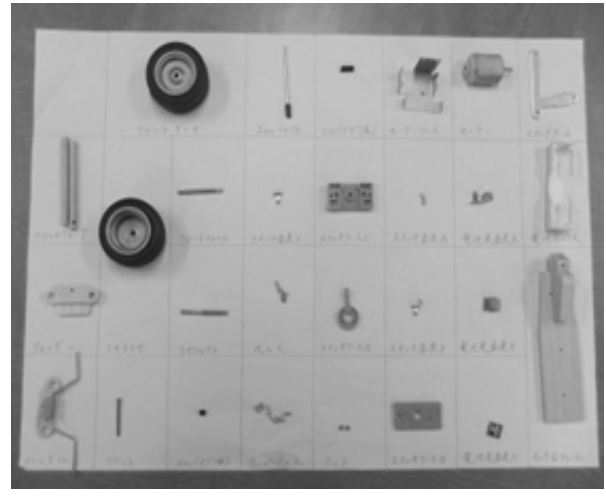

Figure 5. Parts layout for the assembly training experiment.

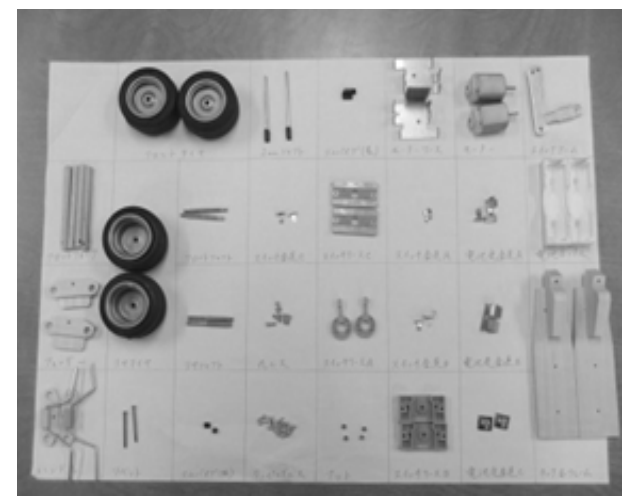

Figure 6. Parts layout for the assembly/disassembly training experiment.

disassembly procedure. This cycle was repeated 10 times, and then the experiment was finished.

All of the subjects were given the instructions similar to the assembly training experiment. 
Table 3. Questions of the work learning confirmation test

\begin{tabular}{cccc}
\hline No. & Problem aspect & The nature of work & Answer \\
\hline 1 & Work procedure & Assembling the rear tire & $\mathrm{F}$ \\
2 & Work procedure & Assembling the front tire & $\mathrm{F}$ \\
3 & Work procedure & Assembling the tapping screw in the battery box & $\mathrm{T}$ \\
4 & Work procedure & Assembling the swing arm & $\mathrm{T}$ \\
5 & Product structure & Assembling the motor case and the switch case & $\mathrm{F}$ \\
6 & Product structure & Assembling the switch fitting & $\mathrm{F}$ \\
7 & Product structure & Assembling the metal fitting & $\mathrm{T}$ \\
8 & Work procedure & Assembling the metal fitting & $\mathrm{F}$ \\
9 & Product structure & Assembling the battery box & $\mathrm{F}$ \\
10 & Product structure & Assembling the tire and the shaft & $\mathrm{F}$ \\
\hline
\end{tabular}

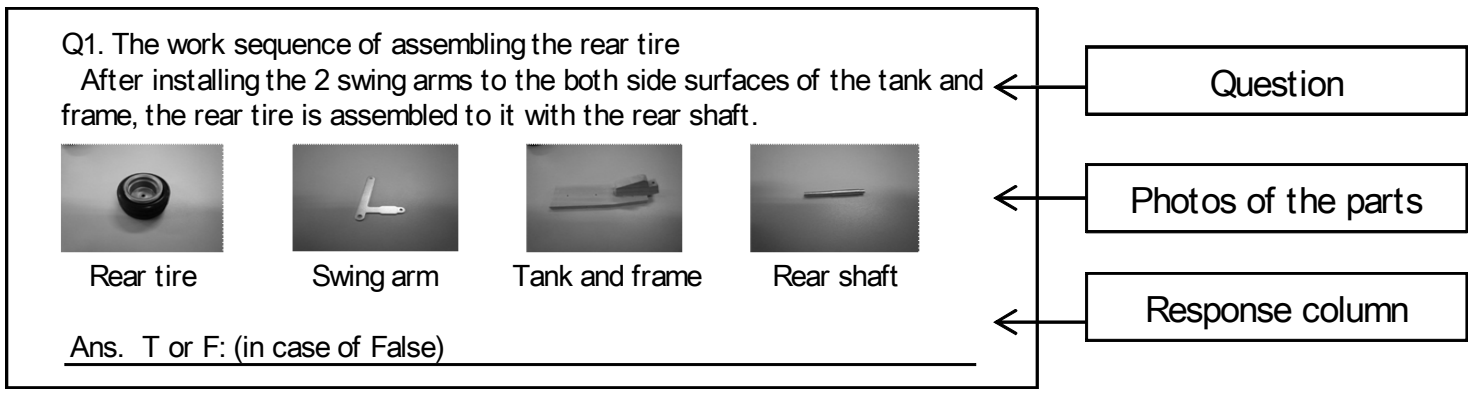

Figure 7. Example of the problem of the work learning confirmation test.

\subsection{Work Learning Confirmation Test}

Subsequent tests were performed for both the assembly training experiment and the assembly/disassembly training experiment.

The work learning confirmation test evaluated the degree of understanding of work content immediately after each training experiment. After the training experiments, each subject was immediately tested with 10 true or false questions. The time limit was set to 10 minutes, and the subjects were asked to raise their hand after answering all 10 questions. At that point, the test was finished. The speed of recollecting the work procedure was evaluated based on the response time, and the accuracy of the learned work procedure content was evaluated based on the number of correct answers.

As shown in Table 3, five questions on the work learning confirmation test pertained to the work procedure, and five questions pertained to the product structure. As in the example shown in Figure 7, a question was presented for each problem, and a photo of the part relating to the question was shown below that. Finally, a response column was provided, and the subjects were asked to choose either true (T) or false (F). When " $F$ " was selected, they were asked to correct the error in the quetion. For example, Question 1 relates to mounting the rear tire, and it confirms memory of the work procedure.

Prior to the test, all of the subjects were given the following instructions besides the answering manner. Firstly, a subject is expected to answer the questions as fast and correctly as possible. Secondly, no questions about the problems are allowed.

\subsection{Post-training Experiment}

The following experiment was conducted on the 7th day from the day the aforementioned experiment was conducted. It was expected that the subjects would tend to forget the motions and the work procedures as the number of days increased since training. It was evaluated experimentally whether there are any differences in retention due to the difference between the assembly training and the assembly/disassembly training.

\subsubsection{Work retention confirmation test}

The work retention confirmation test evaluated the retention of the motions and the work procedures seven days after conducting training experiments. Mini-bike assembly work was performed one time. At that time, the work procedure was not shown to the subjects, but if they could not remember the procedure, they were allowed to ask questions. The speed of motion during work was evaluated based on the work time, and the accuracy of memory of the work procedure was evaluated based on the number of questions. Since it is difficult to directly evaluate the accuracy of motions and the 


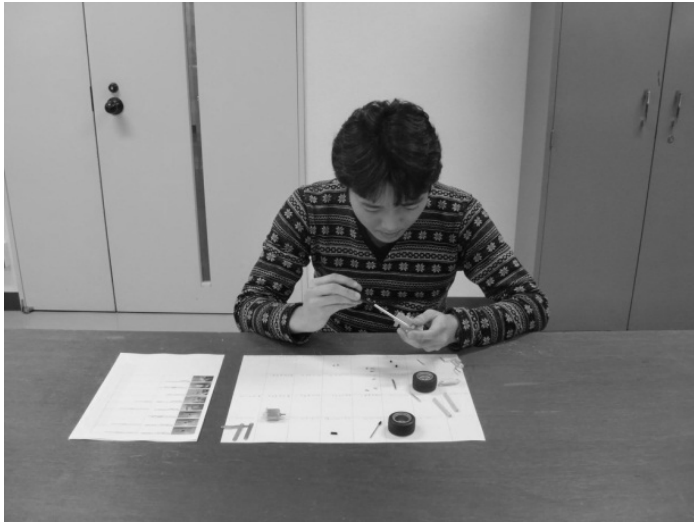

(a)

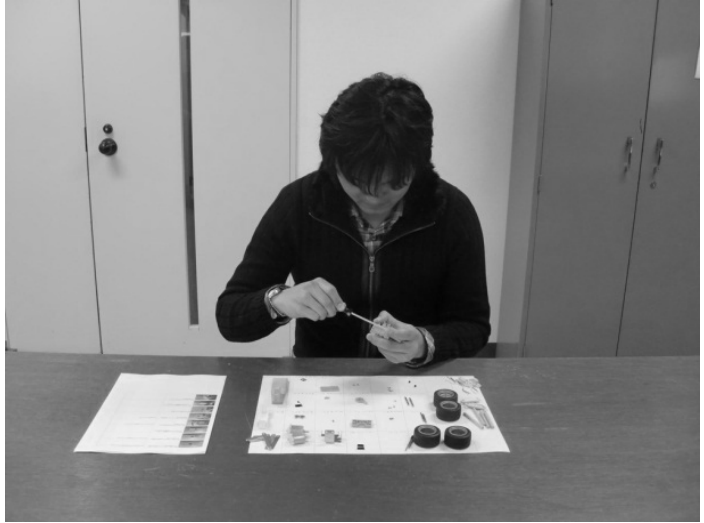

(b)

Figure 8. Scene of experiment. (a) Assembly training experiment and (b) assembly/disassembly training experiment.

Table 4. Results of the grouping experiment

\begin{tabular}{|c|c|c|c|c|c|}
\hline $\begin{array}{l}\text { Subject } \\
\text { no. }\end{array}$ & $\begin{array}{l}\text { Work time } \\
\text { (s) }\end{array}$ & $\begin{array}{l}\text { Total distance of } \\
\text { the gaps }(\mathrm{mm})\end{array}$ & $\begin{array}{l}\text { Recollection } \\
\text { time }(\mathrm{s})\end{array}$ & $\begin{array}{l}\text { The number of } \\
\text { correct answers }\end{array}$ & $\begin{array}{c}\text { Assigned training } \\
\text { experiment }\end{array}$ \\
\hline 1 & 219 & 0.13 & 39 & 16 & \multirow{6}{*}{ Assembly } \\
\hline 2 & 188 & 0.27 & 180 & 11 & \\
\hline 3 & 237 & 2.39 & 51 & 16 & \\
\hline 4 & 237 & 1.57 & 97 & 12 & \\
\hline 5 & 166 & 5.20 & 75 & 13 & \\
\hline 6 & 163 & 5.72 & 119 & 12 & \\
\hline 7 & 184 & 0.93 & 33 & 16 & \multirow{6}{*}{ Assembly/disassembly } \\
\hline 8 & 224 & 2.38 & 40 & 14 & \\
\hline 9 & 224 & 2.20 & 114 & 14 & \\
\hline 10 & 203 & 0.62 & 81 & 12 & \\
\hline 11 & 180 & 1.83 & 57 & 8 & \\
\hline 12 & 227 & 3.92 & 39 & 12 & \\
\hline
\end{tabular}

speed of recollecting learned work procedures, the evaluation was done indirectly based on the number of mistakes and the work time for the cycle.

Prior to the test, all of the subjects were given the following instructions besides the purpose of this test, the state of starting the test, and the state of finishing the test. Firstly, a subject is not allowed to look at the assembly procedure while the experiment. Secondly, when a subject forgets any steps of the work procedure, the subject should raise the hand and ask questions in order to confirm the correct procedure.

\subsubsection{Structure Retention Confirmation Test}

The structure retention confirmation test evaluated the retention of memory relating to product structure seven days after conducting the training experiments. One mini-bike containing 2 types of mistakes was used, and a test where the subjects corrected those mistakes was conducted. The nature of the 2 mistakes was communicated to the subjects beforehand. The 2 types of mistakes were "reversed position for mounting the switch fitting A" and "installation of the battery box with reversed direction." These mistakes were selected because they require the largest number of steps to correct. The recollection speed was evaluated based on the thinking time until the correction method was decided upon, and the accuracy of memory was evaluated based on the number of steps required for correction.

Prior to the test, all of the subjects were given the following instructions besides the purpose of this test, the state of starting the test, and the state of finishing the test. A subject can start to correct either of the 2 mistakes, but both of them must be corrected by the end of the test.

\section{EXPERIMENT RESULTS AND DISCUSSION}

\subsection{Experiment Plan}

In the experiments, 12 university or graduate school students were selected as subjects. First, the grouping experiment was conducted, and the subjects were divided into 2 groups of 6 subjects, based on the results so 


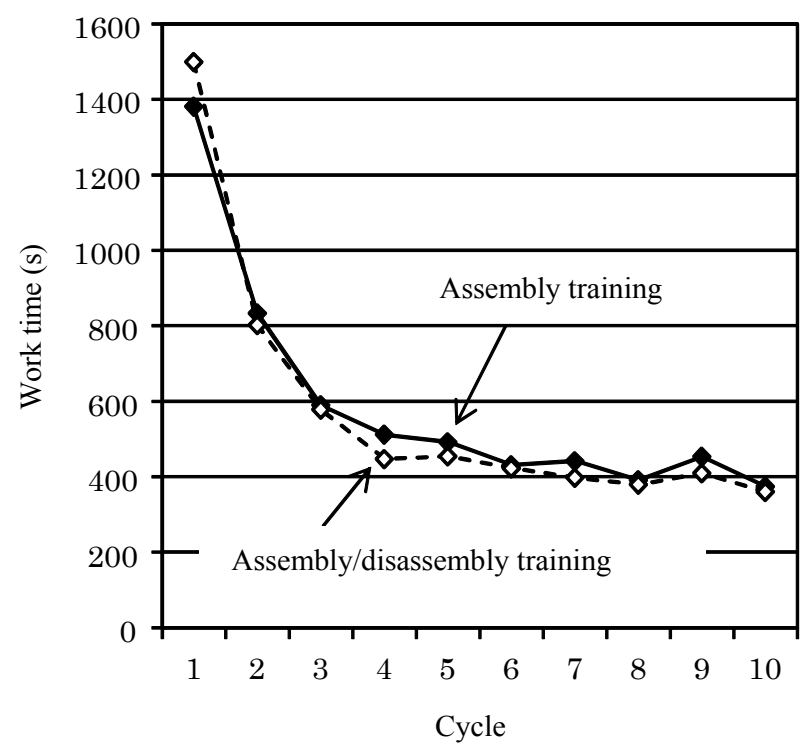

Figure 9. Example of the problem of the work learning confirmation test.

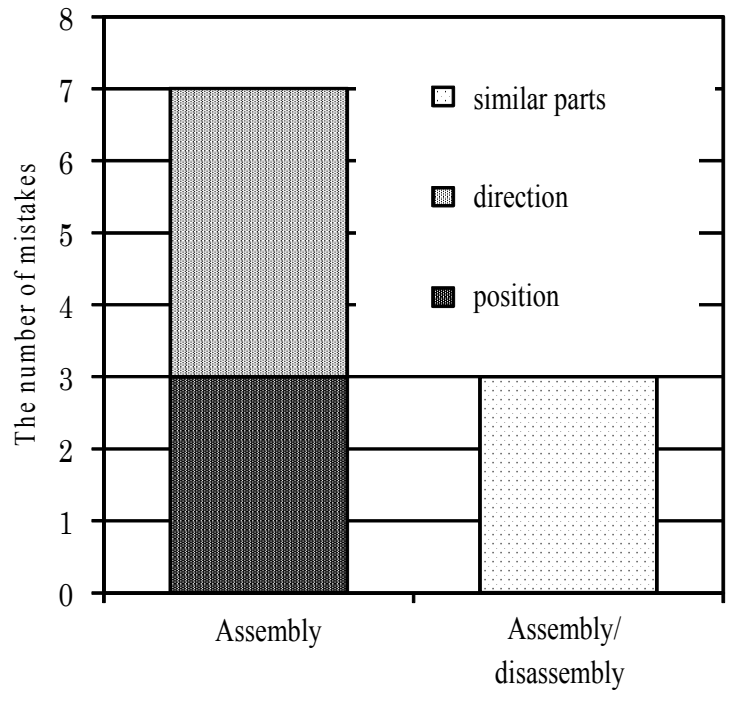

Training experiment

Figure 10. The total number of mistakes in each training experiment. that no differences caused by subject's ability would appear in the subsequent experiments. After that, the training experiments and work learning confirmation test were carried out for each subject. Then, seven days after the experiment, the 2 types of post-training experiments were carried out. Figure 8 shows the scenes of the experiment (the assembly training experiment and the assembly/disassembly training experiment). The experiment environment was maintained at a comfortable room temperature and humidity using air-conditioning equipment. In addition, the subjects were not affected by outside noise.

The results of each experiment are discussed below.

\subsection{Grouping Experiment}

The results of the grouping experiment are shown in Table 4. A correlation diagram was drawn regarding the work time and total distance of the gaps, which were the results of the screw tightening test, and the subjects were ordered starting from the subject with results closest to the origin. Ordering was done in the same way for memory time and number of correct answers from the card arrangement memory test. The far right column of the table shows the results of assigning subjects to the assembly training experiment and the assembly/disassembly training experiment by taking into account the above orderings.

In order to check the difference between subjects assigned to the two training experiments, $\mathrm{F}$ tests and $\mathrm{t}$ tests were conducted on the work time, the total distance of the gaps, the memory time, and the number of correct answers. The results showed no significant difference in any of the cases. The fact that there were no significant differences does not mean that there were no differences between subjects assigned to the two experiments. However, there was no notable difference, and thus in this study it was determined that grouping was effective to a certain extent.

\subsection{Training Experiments}

Figure 9 shows a line graph of the work time (average for all subjects) by cycle in the assembly training experiment and the assembly/disassembly training experiment. Figure 10 shows a bar graph of the total number of mistakes made by all subjects, and the bars are broken down by the type of mistake. No difference was found between the two training experiments in the work time variation. $F$ tests and $t$ tests were conducted for each cycle, and in both cases, there were no cycles with a significant difference. Therefore, it was determined that there would be no differences in the work time due to training.

There were fewer mistakes (3) in the assembly/ disassembly training experiment than in the assembly training experiment (7). When the nature of the mistakes in the assembly training experiment was analyzed, there were 3 position-related mistakes (e.g., mounting parts at the wrong position) and 4 direction-related mistakes (e.g., reversing the mounting direction of parts). It is thought these mistakes occurred due to inadequate memory of the product structure. In contrast, these mistakes did not occur in the assembly/disassembly training experiment. This suggests the possibility that the assembly/disassembly training promotes memory of product structure. In the assembly/disassembly experiment, on the other hand, there were 3 mistakes relating to similar 


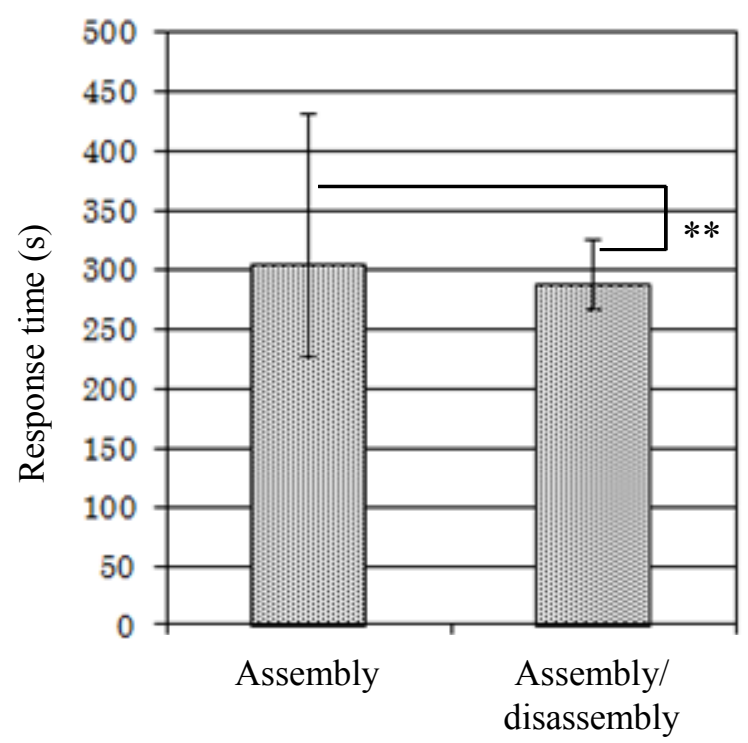

Training experiment

Figure 11. Response time in the work learning confirmation test.

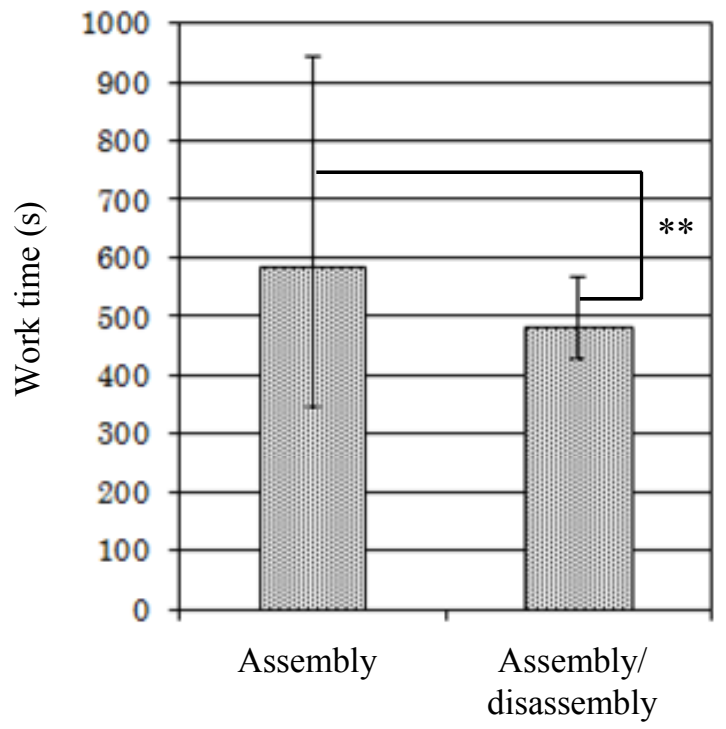

Training experiment

Figure 13. Work time in the work retention confirmation test.

parts (e.g., mistaking round screws with tapping screws), and these mistakes did not occur in the assembly training experiment. Rather than being caused by memory of the structure, it is thought that these mistakes were simply errors. However, the fact that they occurred only in the assembly/disassembly training experiment shows that there may have been an effect due to confusion, resulting from the fact that subjects performed both assembly and disassembly. Caution is needed on this point.

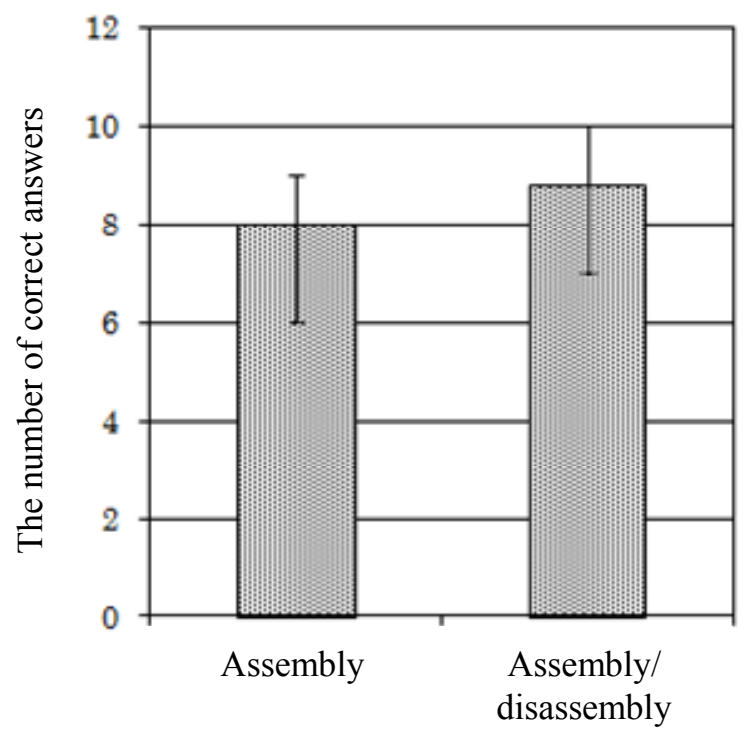

Training experiment

Figure 12. The number of correct answers in the work learning confirmation test.

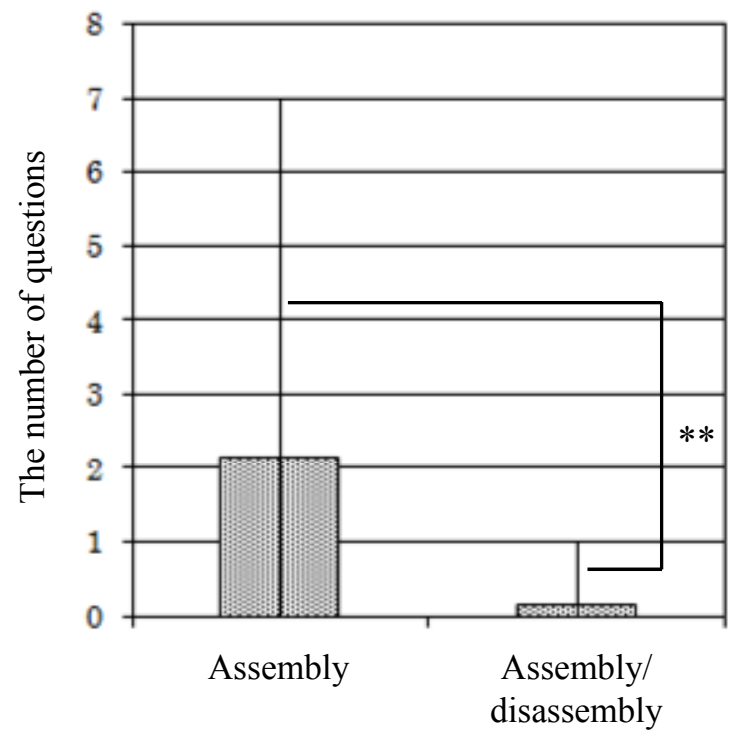

Training experiment

Figure 14. The number of questions in the work retention confirmation test.

\subsection{Work Learning Confirmation Test}

The work learning confirmation test was conducted immediately after the training experiments. For the assembly training experiment and the assembly/disassembly training experiment, Figure 11 shows a bar graph of the response time, and Figure 12 shows a bar graph for the number of correct answers. In both of these graphs, the maximum and the minimum values in each case are 


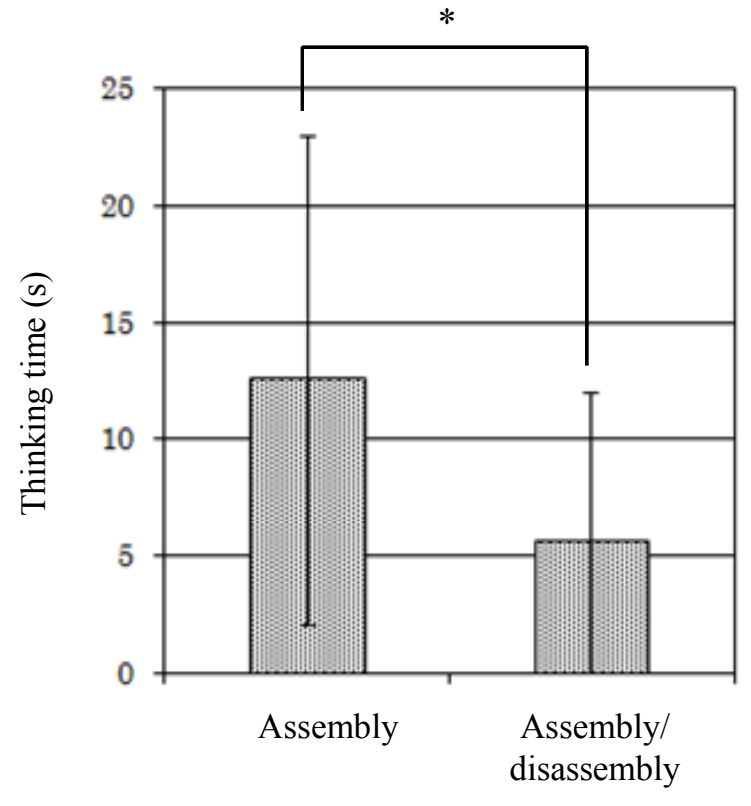

Training experiment

Figure 15. Thinking time in the structure retention confirmation test.

indicated by lines on the bars.

In the graph of the response time, the difference between the maximum and the minimum value is smaller for the assembly/disassembly training experiment than for the assembly training experiment. The results of the $F$ test showed a significant difference at a significance level of $1 \%(\mathrm{~F}(5,5)=14.63)$, and a difference in variance. The average response time was somewhat shorter for the assembly/disassembly training experiment than the assembly training experiment. However, in the results of a one-tailed t test assuming that the variances are not equal, there was no significant difference, and no difference was found in the average values.

In the graph of the number of correct answers, there was no major difference in the differences between maximum and the minimum values, but the average for the assembly/disassembly training experiment is somewhat greater than that for the assembly training experiment. However, the results of the F test and a one-tailed $\mathrm{t}$ test showed no significant difference.

Due to the above results, it was determined that the assembly/disassembly training leads to more stable recollection of work procedures, and it does not have an adverse effect on the accuracy of learning work procedures.

\subsection{Work Retention Confirmation Test}

The work retention confirmation test was conducted seven days after the training experiments. For the assembly training experiment and the assembly/disassembly training experiment, Figure 13 shows a bar graph

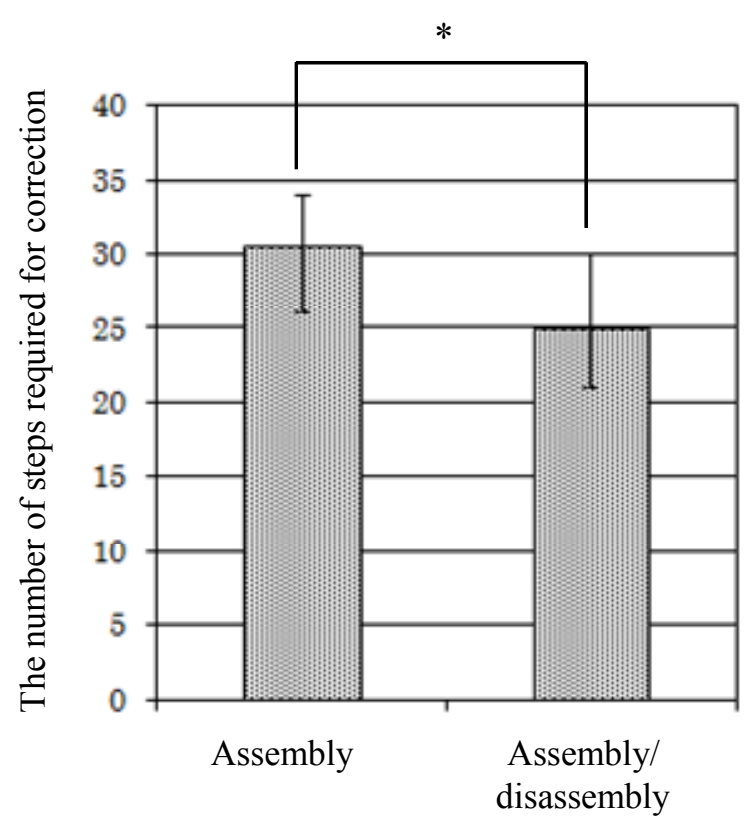

Training experiment

Figure 16. The number of steps required for correction in the structure retention confirmation test.

of the work time, and Figure 14 shows a bar graph of the number of questions. In both of these graphs, the maximum and minimum values in each case are indicated by lines on the bars.

In the graph of the work time, the difference between the maximum and the minimum value is smaller for the assembly/disassembly training experiment than for the assembly training experiment. The results of the $F$ test showed a significant difference at a significance level of $1 \%(F(5,5)=18.99)$, and a difference in variance. The average work time was shorter for the assembly/disassembly training experiment than the assembly training experiment. However, in the results of a onetailed $\mathrm{t}$ test assuming that the variances are not equal, there was no significant difference, and no difference was found in the average values.

In the graph of the number of questions, the difference between the maximum and the minimum value is smaller for the assembly/disassembly training experiment than for the assembly training experiment. The results of the $\mathrm{F}$ test showed a significant difference at a significance level of $1 \%(F(5,5)=49.00)$, and a difference in variance. The average number of questions was smaller for the assembly/disassembly training experiment than for the assembly training experiment, and was almost 0 . However, in the results of a one-tailed t test assuming that variances are not equal, there was no significant difference, and no difference was found in the average values.

Due to the above results, it was determined that the assembly/disassembly training leads to more stable retention of the motions and the work procedure seven 
Table 5. Results of the experiments

\begin{tabular}{|c|c|c|c|c|c|c|c|c|}
\hline \multirow{3}{*}{ Experiment } & \multicolumn{4}{|c|}{ Motion } & \multicolumn{4}{|c|}{ Work procedure } \\
\hline & \multicolumn{2}{|c|}{ Speed } & \multicolumn{2}{|c|}{ Accuracy } & \multicolumn{2}{|c|}{ Speed } & \multicolumn{2}{|c|}{ Accuracy } \\
\hline & Ave. & Var. & Ave. & Var. & Ave. & Var. & Ave. & Var. \\
\hline \multicolumn{9}{|l|}{ Undergoing training } \\
\hline Training experiment & - & - & - & - & - & - & - & - \\
\hline \multicolumn{9}{|l|}{ Immediately after training } \\
\hline Work learning confirmation test & & & & & - & $* *$ & - & - \\
\hline \multicolumn{9}{|l|}{ Seven days after training } \\
\hline Work retention confirmation test & - & $* *$ & - & $* *$ & - & $* *$ & - & $* *$ \\
\hline Structure retention confirmation test & & & & & $*$ & - & $*$ & - \\
\hline
\end{tabular}

-: no significant, $*$ : significant difference at a significance level of $5 \%, * *$ : significant difference at a significance level of $1 \%$.

days after training.

\subsection{Structure Retention Confirmation Test}

The structure retention confirmation test was conducted seven days after the training experiment, after the above training. For the assembly training experiment and the assembly/disassembly training experiment, Figure 15 shows a bar graph of thinking time, and Figure 16 shows a bar graph of the number of correction steps. In both of these graphs, the maximum and the minimum values in each case are indicated by lines on the bars.

In the graph of the thinking time, the difference between the maximum and the minimum value is somewhat smaller for the assembly/disassembly training experiment than for the assembly training experiment. However, the results of the $\mathrm{F}$ test showed no significant difference. The average thinking time in the assembly/disassembly training experiment was less than half that of the assembly training experiment. The results of a one-tailed t test showed a significant difference at a significance level of $5 \%(\mathrm{t}(10)=1.89)$, and a shorter thinking time for the assembly/disassembly training experiment.

In the graph of the number of correction steps, the difference between the maximum and the minimum values is about the same for both experiments, and the results of the $\mathrm{F}$ test showed no significant difference. The average value of the number of correction steps was smaller for the assembly/disassembly training experiment than for the assembly training experiment. The results of a one-tailed $t$ test showed a significant difference at a significance level of $5 \%(t(10)=2.65)$, and a shorter thinking time for the assembly/disassembly training experiment.

Due to the above results, it was determined that the assembly/disassembly training is more effective for retention of structure memory seven days after training.

\subsection{Summary of Experiment Results}

The above experimental results are summarized in
Table 5. In this table, “-” indicates experimental results where there was no significant difference; "*” indicates cases where there was a significant difference at a significance level of $5 \%$; and "**" indicates cases where there was a significant difference at a significance level of $1 \%$. The table also shows the pertinent results for the indirect evaluation in Section 4 (area of the table with light shading).

It was shown that, during training, the assembly/disassembly training may be more effective than the assembly training for memory of structure, but there was no major difference between the two types of training. However, the assembly/disassembly training was more effective than the assembly training in terms of variance in the speed of recollecting the work procedure. That is, the assembly/disassembly training is more stable than the assembly training in terms of the time for remembering the work procedure.

Seven days after training, there was no difference in the speed of either motion or recollection, but in terms of variance, the assembly/disassembly training was more effective than the assembly training. That is, the assembly/disassembly training was more stable in terms of learning the motions and the work procedure even seven days after training. With regard to memory of product structure, the thinking time was shorter, and the number of steps needed to perform correction was smaller for the assembly/disassembly training than for the assembly training. That is, compared to the assembly training, the assembly/disassembly training promotes memory of product structure, and the effect can be maintained even seven days after training.

For the above reasons, it was determined that the experiments verified the effectiveness of the assembly/ disassembly training proposed in this study.

\section{CONCLUSION}

The aim of this study was to propose a work training method employing disassembly, carried out by the trainer during training, assuming a product with a great 
deal of manual assembly such as office machines. The assembly/disassembly training was devised, in which the workers undergoing training perform disassembly work, as a contrast to the assembly training where the trainer performs disassembly work. To verify the effectiveness of this new approach, an experiment using assembly work for the mini-bike was designed, and then experimental analysis was carried out regarding speed and accuracy of motion, and speed and accuracy of learning the work procedure (recollection) at three time pointsduring training, immediately after training, and seven days after training. As a result, it was possible to confirm that, compared to the assembly trainning, the assembly/disassembly training improves stability of work procedure recollection immediately after training, improves retention of the motions and the work procedure from the time of training to seven days after training, and promotes and maintains memory relating to product structure. As a result of the above, the experiments showed the effectiveness of the assembly/disassembly training method proposed in this study.

In the future, the authors plan to continue this research by, among other things, expanding it to assembly work of products other than the mini-bike, and further improving the effectiveness of the assembly/disassembly training.

\section{REFERENCES}

Alchian, A. (1963), Reliability of progress curves in airframe production, Econometrica, 31(4), 679-693.

Bevis, F. W., Finnlear, C., and Towill, D. R. (1970), Prediction of operator performance during learning of repetitive tasks, International Journal of Production Research, 8(4), 293-305.

Fujita, A. (1978), The Fundamentals on IE, Kempakusha, Tokyo, Japan.

Fukuda, Y. and Ohkubo, A. (1993), A study on learning of some works by work achievement method, $\mathrm{Ja}$ panese Journal of Ergonomics, 29(4), 215-222.

Hirschmann, W. B. (1964), The learning curve, Chemical Engineering, 71(7), 95-100.

Ichikizaki, O. (2006), A fundamental study on mistakes made by workers who have acquired the work procedure, Japanese Journal of Ergonomics, 42(3), 172-180.

Ichikizaki, O., Kanazawa, T., Cho, H., and Yamazaki, T. (2009), A fundamental study on analysis of acqui- sition process of work sequence and positions, Proceedings of the Asia Pacific Industrial Engineering and Management Systems Conference, Kitakyushu, Japan, 1714-1723.

Ichikizaki, O., Yamazaki, T., and Kanazawa, T. (2010), A fundamental study on influence of errors on acquisition of work procedure in the acquisition process, Japanese Journal of Ergonomics, 46(6), 362372.

Jittachalothorn, P. (1998), A study on memorization process of work sequence, Japanese Journal of Ergonomics, 34(3), 129-142.

Jittachalothorn, P. (2000), A study on memorization advancement method of work sequence, Japanese Journal of Ergonomics, 36(3), 105-117.

Jittachalothorn, P., Matsumoto, T., and Kanazawa, T. (1996), A study on learning process using information memory and evaluation method, Japanese Journal of Ergonomics, 32(2), 63-70.

Jittachalothorn, P., Matsumoto, T., and Kanazawa, T. (1997), A study on the effect of kinds and number of assembled parts on the memorization method in learning process, Japanese Journal of Ergonomics, 33(5), 305-315.

Seibel, R. (1964), Levels of practice, learning curves, and system values for human performance on complex tasks, Human Factors, 6(3), 293-298.

Shida, K. (2005), A basic study on motion pictures for the work instruction using a personal computer in an assembly work, Japanese Journal of Ergonomics, 41(1), 1-10.

Shida, K., Ichikizaki, O., Matsumoto, T., and Kanazawa, T. (2003), A basic study on presentation information using a personal computer for the work instruction in assembly work: a comparison of the character information and the still picture information, Japanese Journal of Ergonomics, 39(5), 219231.

Suenaga, O. (1998), A study on training using active controller in man-machine system, Japanese Journal of Ergonomics, 34(2), 67-77.

Suenaga, O. and Ihara, M. (1995), A study on training using active controller in man-machine system, Japanese Journal of Ergonomics, 31(1), 21-29.

Wright, T. P. (1936), Factors affecting the cost of airplanes, Journal of the Aeronautical Sciences, 3(4), 122-128. 